\title{
Research Progress of Hysteroscopic Technology
}

\author{
Xiaojun Liu ${ }^{1}$, Jiawen Zhou* ${ }^{1}$ \\ ${ }^{1}$ China-Japan Union hospital of Jilin University, Changchun, Jilin, 130033
}

Keywords: research progress; Hysteroscopic technology; medical application; control charts, process capability

\begin{abstract}
Because hysteroscopy diagnosis is intuitive and accurate with other characteristics and advantages, for the treatment of benign diseases such as abnormal uterine bleeding and submucosal fibroids, hysteroscopic surgery is curable. Hysteroscopy is very important in the diagnosis and treatment of gynecological diseases. This paper summarizes the progress, status and role of hysteroscopic treatment in recent years.
\end{abstract}

\section{Introduction}

As an important part of gynecological minimally invasive surgery, hysteroscopy has become increasingly popular in clinical applications. The renewal and improvement of equipment and equipment make the surgical process more simple, and the maturity of operation skills makes the surgical indications continue to widen. As a micro-trauma surgical method under direct vision, hysteroscopy has been widely involved in the diagnosis and treatment of uterine diseases, and with its Intuitive and accurate, invasive, able to restore the anatomy of the uterine cavity, replace the hysterectomy to cure abnormal uterine bleeding and other advantages, has become an indispensable tool for clinical diagnosis and treatment of gynecology. It is proposed to discuss the clinical application of hysteroscopic surgery, the treatment of more common problems, the disputes in hysteroscopic intervention in the diagnosis and treatment of uterine malignancy, and the treatment of common operative complications in hysteroscopic surgery.

\section{Status and Development Trend of Hysteroscope Clinical Application}

Hysteroscopy can be directly viewed and can be located for histological examination, the accuracy of its diagnosis has been confirmed by a large number of clinical studies. In recent years, new types of hysteroscopes have been continuously developed, and hysteroscopes that are designed and constructed to conform to the anatomy of the uterus and microsurgical hysteroscopes with an access hole have been used clinically. The external diameter of the insertion end of the fiber hysteroscope scope is only 2.9 to $3.1 \mathrm{~mm}$, and it can be bent up and down from $100^{\circ}$ to $120^{\circ}$. It is easy to observe the internal appearance of the uterine cavity through the cervical canal, even for the non-birth or postmenopausal women without anesthesia. Under state; fiber hysteroscope slim and flexible mirror body structure is also easy to pass the young girl or unmarried adult woman's hymen into the vagina to the cervix, even through the cervix into the uterine cavity, the intrauterine lesions were examined directly. Miniature hard-tube hysteroscopes can insert micro-surgical instruments such as grasping forceps, scissors, tubal catheters, etc. to perform intrauterine operation and fallopian tube dredge treatment through the operation holes on the sheath. The renewal and improvement of equipment and equipment make the operation process simpler, no need for anesthesia or only local anesthesia, no need to dilate the cervix, and hysteroscopy diagnosis and diagnosis that can perform surgical operation at the same time can be implemented in the outpatient department, which not only simplifies the procedure for diagnosis and treatment, but also saves The patient's diagnosis and treatment costs. The coaxial bipolar micro-hysteroscope developed and researched by GYNECARE Inc. is a combination of diagnostic and therapeutic intervention. Electrolyte liquid is allowed to be used as a uterine distention medium during operation, which reduces the surgical complications caused by excessive absorption of non-electrolyte media to some 
extent. Marwah reported that outpatient treatment was performed on 151 patients through coaxial bipolar hysteroscopy. At the time of diagnosis, the patient included isolation of intrauterine adhesions, removal of endometrial polyps, resection of submucous myoma, oviduct intubation, and uterus Surgical treatment, including excision and endometrial removal, achieved satisfactory clinical efficacy, and all patients were discharged during surgery. Mini hysteroscopy can complete most intrauterine surgical procedures. Bettocchi reported using a $5 \mathrm{~F}$ hysteroscope with an access hole for the diagnosis of 4,863 patients undergoing surgical treatment of endometrial polyps (polypoid diameter 0.2 to $3.7 \mathrm{~cm}$ ), intrauterine adhesions, and other uterine cavity abnormalities. None of the anesthetic drugs were used. $71.9 \%$ to $93.5 \%$ of the patients were completely tolerant of surgery, indicating that the micro-devices make clinic hysteroscopy treatment safer and more feasible.

Endometrial polyps, submucosal fibroids and endometrial lesions are common causes of abnormal uterine bleeding (AUB). Traditional surgical treatment is hysterectomy. In the United States, about 750000 cases of hysterectomy are performed each year, of which more than $60 \%$ are AUB, and the rate of complications in various types of surgery is as high as $30 \%$ to $40 \%$. Hysteroscopic surgery for abnormal bleeding caused by intrauterine lesions has its unique advantages, without the need to open the abdomen and resection of the uterus, is a minimally invasive surgical method to replace AUB treatment of hysterectomy. Hysteroscopic treatment of endometrial lesions caused by AUB is based on the destruction of the entire layer of the endometrium and underlying muscle tissue, prevent intimal regeneration, to amenorrhea or reduce menstruation purposes. We summarized the experience of transcervical resection of endometriosis (TCRE) in patients with dysfunctional uterine bleeding (DUB) who was ineffective for drug treatment, required retention of uterus, and no reproductive requirements. The average follow-up was 71.42 . \pm 23.33 months, the total effective rate of menstrual improvement was $94.5 \%$. Among them, the rate of menopause was $25.8 \%$, the rate of menstruation decreased was $68.7 \%, 97.6 \%$ of patients with preoperative combined anemia were corrected, and more than $90 \%$ of patients were satisfied with the effect of treatment. A large number of multi-center clinical studies abroad have also shown that TCRE has opened up a new surgical treatment for DUB.

\section{Clinical Application and Precautions for Hysteroscopic Treatment and Transection}

Hysteroscopic tubal intubation and injection of drugs to clear the fallopian tube can be used for infertility patients confirmed tubal interstitial obstruction, or after repeated tubal fluid and tubal lipiodol angiography, diagnosis of tubal adhesion infertility. Dysfunctional uterine bleeding (DUB) hysteroscopic endometrial transurethral resection of DUB is based on the destruction of the entire endometrium and underlying muscular tissue, preventing endometrial hyperplasia, reaching amenorrhea or reducing menstruation effect. A study from a multi-centered medical institution in the UK showed that hysteroscopic endometrial ablation (TCRE) opened up a new surgical treatment for DUB. DUB patients with fertility requirements are not eligible for TCRE treatment.

Endometrial polyps and submucosal myomas hysteroscopic endometrial polypectomy (TCRP) and hysteroscopic myomectomy (TCRM) do not disrupt normal uterine anatomy, can cure abnormal uterine bleeding, and improve the patient's reproductive prognosis. Resection of the basal layer by TCRP can prevent its persistence and recurrence. TCRP and TCRM should be preoperatively ruled out for malignant transformation, with particular emphasis on TCRM procedures in combination with the surgeon's skill level, clinical experience, and patient specificity to determine indications for surgical adaptation. For diameters $\geq 5 \mathrm{~cm}$, multiple, wide pedicles, and intermural fibroids, Before surgery, preconditioning should be considered to reduce fibroids and uterine volume, to reduce the difficulty of surgery, and to avoid intraoperative complications. For submucosal myoma of deep submucosa, the first surgery removes fibroids and peripheral muscles, and the remaining part is to be implanted afterwards. Reoperation in the uterine cavity.

Hysteroscopic surgery to separate intrauterine adhesions (TCRA) is a uterine cavity reconstructive surgery that can restore the normal anatomy of the uterine cavity, correct menstrual abnormalities and regain fertility in patients with infertility. More used in uterine septal 
malformations. Xia Enlan [6] reported that 116 cases of uterine septum treated by hysteroscopic uterine septum resection (TCRS) were effective, and provided a new surgical choice for the treatment of uterine dysplasia.

Cervical benign lesion resection for patients with recurrent cervical polyps, symptomatic cervical polypoid hyperplasia, cervical erosion treatment failure or healing defects, intracervical oncology grade I II patients. The incidence rate was $0.45 \%$ to $4 \%$. The main sites of proneness were in the uterine horn, fundus, isthmus, etc. The incidence and anatomy, the type of operation (TCRA, TCRM, TCRS), the experience of the surgeon, the previous uterus History of surgery and so on. After the occurrence of uterine perforation, the site and extent of perforation should be determined immediately to determine the amount of bleeding and whether there are any other organ injuries, and a treatment plan should be established according to the specific situation. Venous air embolism or venous gas embolism is a serious, rare but fatal complication of surgery. Therefore, monitoring should be strengthened during surgery, including continuous precordial Doppler monitoring, end-tidal $\mathrm{CO} 2$ pressure monitoring and blood oxygen saturation measurement. The incidence of acute air embolism is critical and it is difficult to save. Clinical prevention is the mainstay.

Although a large number of clinical studies at home and abroad have fully affirmed the value of hysteroscopy in endometrial lesions, especially in the diagnosis of early endometrial cancer, hysteroscopy requires proper perfusion media (liquid, gas) and uterine distention pressure. Expansion of the uterine cavity, perfusion media in the expansion of the uterine cavity, flushing of the intimal debris and blood clots to provide a clear observation of the field of vision, but also will make part of the endometrium debris and other intrauterine contents into the peritoneal cavity through the open fallopian tube. Whether hysteroscopy can cause the proliferation of endometrial cancer cell peritoneal cavity and the proliferation of endometrial cancer cells increase the survival risk of patients with endometrial cancer? This year, Professor Wang Hongwu, Department of Gynecologic Oncology, Peking University Hospital will elaborate on this. In recent years, the general trend of hysteroscopy and endometrial cancer spread is that hysteroscopy may cause endometrial/endometrial cancer cells to spread in the peritoneal cavity, but disseminated endometrial cancer cells do not affect patients. The survival prognosis.

\section{Minimally Invasive Techniques Such as Laparoscopic Diagnosis and Treatment of Gynecological Diseases Combined}

Hysteroscopic laparoscopic surgery can be used to diagnose and treat two or more diseases in the uterine cavity and intra-abdominal cavity simultaneously under one anesthesia. Uterine cavity reconstruction and reconstructive surgery and large TCRM fibroids, laparoscopic monitoring can directly observe changes in the serosal surface of the uterus, prevent and detect uterine perforation, and can also repair damaged organs at the same time. Laparoscopic light transmission test can indicate whether the septum resection and intrauterine adhesion separation are in place and cut. Uterine cavity reconstruction and reconstructive surgery and large TCRM fibroids, laparoscopic monitoring can directly observe changes in the serosal surface of the uterus, prevent and detect uterine perforation, and can also repair damaged organs at the same time. Laparoscopic light transmission test can indicate whether the septum resection and intrauterine adhesion separation are in place and cut.

Hysteroscopic resection of submucosal and/or intermural endomyoid tumors, laparoscopic resection of subserosa, extrapancreatic and/or uterine fibroids, laparoscopic suturing of fibroid basal and muscular walls, embedding of serosa Floor. Laparoscopic hysteroscopic tubal intubation methylene blue liquid, can directly observe the flow of methylene blue from the unobstructed fallopian tube umbrella, such as obstruction of the fallopian tube, you can see the blocked area, select the treatment program, but also help To understand the shape of the fallopian tube, diagnosis and treatment of pelvic adhesions, endometriosis and other diseases that impede pregnancy. It has been reported in the literature that hysteroscopic laparoscopy combined with tubal intubation has achieved reoperation rates of $70 \%$ to $92 \%$. The postoperative follow-up time was more than 12 months. The intrauterine pregnancy rate was $47 \%$ and the ectopic pregnancy rate was 8 . \%. For type 
II or intermural fibroids with a diameter greater than or equal to $5 \mathrm{~cm}$, uterine artery embolization (uterine artery embolization, U AE) blocking uterine blood supply, or hyperthermia focused ultrasound hyperthermia can reduce fibroids Volume, reduce the difficulty of TC RM, increase the chance of a net cut. Hysteroscopic resection after U AE 10. 5 W Cervical pregnancy has also been reported.

With the continuous development and needs of clinical teaching and scientific research technology, hysteroscopy and surgery are becoming safer, more effective, and simpler in microsurgery. The indications will also continue to grow and expand in order to be applied to a wider range of fields. Benefit the majority of women patients. At present hysteroscopy technology still has some problems, such as perfusion system and perfusate, and the power system used in surgery. This will become an important topic for future research, with a view to improving the safety of hysteroscopic surgery and reducing complications. Making a greater contribution to make this minimally invasive surgical technique safer, more effective and popularized.

\section{Conclusion}

In recent years, considerable progress has been made in the development of hysteroscopy, which has replaced traditional open surgery. With the continuous development of science and technology, the continuous development of new medical devices, and the continuous maturation of surgical techniques, hysteroscopy in gynecological clinics will surely have a wider application prospect.

\section{References}

[1] Granberg S, Wikland M, Karlsson B, et al. Endometrial thickness as measured by endovaginal ultrasonography for identifying endometrial abnormality [J]. Am J ObstetGynecol, 1991, 164(1pt 1): 47-52.

[2] Romer T, Schwesinger G. Hormonalinhibition of endometrium for transcervical endometrial ablation-a prospective study with a 2-year follow-up[J]. Eur J Obstet Gynecol ReprodBiol, 1997, 74 (2): 201.

[3] Revel A, Shushan A. Investigation ofthe infertile couple: hysteroscopy withendometrial biopsy is the goldstandard investigation for abnormal uterine bleeding [J]. Hum Reprod, 2002, 17(8): 1947-1949.

[4] Dotto J, Lema B, Dotto JE Jr, et al. Classification of microhysteroscopicimages and their correlation with histologic diagnosis[J]. J Am Assoc Gynecol Laparosc, 2003, 10 (2): 233-246.

[5] Westin B. Hysteroscopy in 2001: acomprehensive review [J]. ActaObstet Gynecol Scand, 2002, 81(7): 681-687. 